\section{Conventional papillary thyroid carcinoma: effects of cystic changes visible on ultrasonography on disease prognosis}

\author{
Ja Young Kim¹, Eun-Kyung Kim¹, Hye Sun Lee², Jin Young Kwak \\ ${ }^{1}$ Department of Radiology, Research Institute of Radiological Science, ${ }^{2}$ Department of \\ Biostatistics, Yonsei University College of Medicine, Seoul, Korea
}

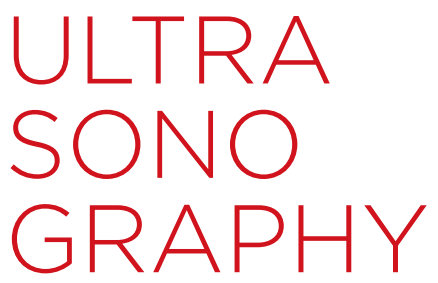

ORIGINAL ARTICLE

http://dx.doi.org/10.14366/usg. 14028 pISSN: 2288-5919 - elSSN: 2288-5943 Ultrasonography 2014;33:291-297

Received: June 25, 2014

Revised: August 1, 2014

Accepted: August 11, 2014

Correspondence to: Jin Young Kwak, MD, Department of Radiology, Yonsei University College of Medicine, 50 Yonsei-ro, Seodaemungu, Seoul 120-752, Korea

Tel. +82-2-2228-7400

Fax. +82-2-393-3035

E-mail: docjin@yuhs.ac
This is an Open Access article distributed under the terms of the Creative Commons Attribution NonCommercial License (http://creativecommons.org/ licenses/by-nc/3.0/) which permits unrestricted noncommercial use, distribution, and reproduction in any medium, provided the original work is properly cited.

Copyright (C) 2014 Korean Society of Ultrasound in Medicine (KSUM)

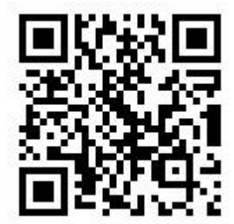

How to cite this article:

Kim JY, Kim EK, Lee HS, Kwak JY. Conventional papillary thyroid carcinoma: effects of cystic changes visible on ultrasonography on disease prognosis. Ultrasonography. 2014 Oct;33(4):291-297. 
Cystic PTC may differ from a cystic change of papillary carcinoma on US. Cystic PTC can manifest as either cystic or solid masses on US [9]. This imaging-pathologic discordance-a mass appearing solid on US, but diagnosed as cystic PTC on pathology-is due to cystic changes within the mass that are too small to be detected on US [9]. Although we often come across PTCs with a cystic appearance on US, there have been no studies on the outcome of PTC showing cystic contents. In this study, we investigated the characteristics of PTC with cystic changes visible on US, comparing them to those showing a solid appearance on US.

\section{Materials and Methods}

\section{Study Population}

This retrospective study was approved by the Institutional Review Board of a Severance Hospital and required neither patient approval nor informed consent for the review of patients' images and medical records. Between January 2003 and August 2004, a total of 923 consecutive patients underwent surgery for thyroid cancer at our institution. Among them, 888 patients were pathologically confirmed as having conventional PTC. Patients were excluded from this study for the following reasons: (1) no available preoperative US images ( $n=176)$, (2) coexisting cancer at another primary site that could affect the prognosis $(n=39)$, (3) previous history of thyroid surgery due to thyroid cancer or recurrent lesions $(n=98)$, (4) no available or insufficient pathology reports $(n=11)$, or (5) follow-up loss after surgery without any available clinical records $(n=11)$. The largest lesion was selected in patients with multiple PTC to avoid data clustering. Finally, this study included 553 thyroid nodules in 553 patients. Seventy of them were men (12.7\%; mean age, 45.6 years; range, 22 to 74 years) and 483 were women (87.3\%; mean age, 45.9 years; range, 17 to 79 years). The mean age of the 553 patients was 45.9 years (range, 17 to 79 years). Detailed characteristics of the patients of this study are presented in Table 1.

The clinical records and pathologic reports were reviewed by two radiologists. Patients were followed up for a mean period of 87.3 months. All included patients underwent total/near-total thyroidectomy with bilateral central neck lymph node dissection (CCND) or subtotal thyroidectomy/hemithyroidectomy with unilateral CCND. Sixty-four patients also underwent lateral neck lymph node dissection for metastatic lymph nodes. Two hundred ninety-two patients underwent subsequent radioactive iodine ablation after thyroidectomy for adjuvant therapy.

Pathology reports were reviewed on the basis of the findings of the nodule size, extrathyroidal extension, multiplicity, lymph node metastases, and underlying thyroid disease. Extrathyroidal extension was categorized into perithyroidal soft-tissue extension, skeletal muscle invasion, or massive extension into the trachea, larynx, esophagus, and recurrent laryngeal nerve. Metastatic lymph nodes were categorized according to location as central and lateral neck lymph nodes. Multiplicity of the thyroid included both multifocality (one lobe) and bilaterality (both lobes) of PTCs.

\section{Ultrasonographic Analysis}

All patients underwent preoperative US examinations. One radiologist (JYK) with 10 years of experience in thyroid imaging retrospectively reviewed the preoperative US images. HDI 3000 or 5000 scanners (Phillips, Bothell, WA, USA) using a bandwidth of a 7- to 12-MHz transducer was used in patients who had US examinations performed at our institution. Twenty-five patients had US examinations done in outside hospitals. These patients were included in this study because the US images obtained were available and determined to be of good quality. The composition of the cancer on US was divided into two categories (less than a $25 \%$ or $50 \%$ cystic component) according to the percentage of cystic components within the cancer lesions.

\section{Postoperative Follow-up}

Follow-up clinical examinations were performed every 6 months during the first 3 years after surgery, and annually thereafter. Blood

Table 1. Detailed demographics of the 553 patients included

\begin{tabular}{lc}
\hline \multicolumn{1}{c}{ Variable } & No. (\%) \\
\hline Age (yr) & $45.9 \pm 12.4$ \\
Mean \pm SD & $45(37-54)$ \\
Median (interquartile range) & $483: 70$ \\
Gender (female:male) & \\
Surgery & $374(67.6)$ \\
Total or near-total & $65(11.8)$ \\
Subtotal & $114(20.6)$ \\
Hemithyroidectomy & \\
Pathologic size (mm) & $12 \pm 9.3$ \\
Mean $\pm S D$ & $10(5-15)$ \\
Median (interquartile range) & \\
Extrathyroidal extension & $321(58)$ \\
No & $232(42)$ \\
Yes & $241(43.6)$ \\
Central lymph node metastasis & $64(11.6)$ \\
Lateral lymph node metastasis & $89(16.1)$ \\
Underlying Hashimoto thyroiditis & $145(26.2)$ \\
Multiplicity & $292(52.8)$ \\
Radioactive iodine ablation & $56(10.1)$ \\
Recurrence &
\end{tabular}


analysis of serum thyroid stimulating hormone (TSH), free thyroxine, thyroglobulin (Tg), and anti-Tg antibody were included in the routine follow-up examination every 12 months. TSH-suppressed serum Tg measurement was routinely used in the follow-up of patients with thyroid cancer. In some patients, stimulated Tg values were used on the basis of a physician's decision. Neck US examinations were also performed routinely. ${ }^{18} \mathrm{~F}$-Fluorodeoxyglucose positron emission tomography with computed tomography, ${ }^{131}$-iodine whole body scan (WBS), or neck computed tomography examinations were performed, selectively. The follow-up interval of patients was clinically defined and calculated as the time from the initial thyroid operation to the most recent clinical evaluation.

Cancer recurrence was evaluated by reviewing all available examinations for each patient. Recurrence was conclusively confirmed by (1) cytopathology obtained from US-FNA and surgical excision, (2) elevated serum $\mathrm{Tg}$, and (3) abnormal uptake

Table 2. Clinicopathologic characteristics of the 553 patients between the two different groups

\begin{tabular}{|c|c|c|c|c|c|c|}
\hline \multirow{2}{*}{ Characteristic } & \multicolumn{3}{|c|}{$50 \%$ Cystic component } & \multicolumn{3}{|c|}{$25 \%$ Cystic component } \\
\hline & $\leq 50 \%(n=546)$ & $>50 \%(n=7)$ & P-value & $\leq 25 \%(n=530)$ & $>25 \%(n=23)$ & P-value \\
\hline Age (yr) & & & 0.825 & & & 0.883 \\
\hline Mean \pm SD & $45.9 \pm 12.4$ & $44.9 \pm 10.8$ & & $45.9 \pm 12.3$ & $46.3 \pm 14.5$ & \\
\hline Median (interquartile range) & $45(37-54)$ & $46(37-56)$ & & $45(37-54)$ & $43(35-58)$ & \\
\hline Gender & & & 0.047 & & & 0.058 \\
\hline Female & $479(87.7)$ & $4(57.1)$ & & $466(87.9)$ & $17(73.9)$ & \\
\hline Male & $67(12.3)$ & $3(42.9)$ & & $64(12.1)$ & $6(26.1)$ & \\
\hline Surgery & & & 0.479 & & & 0.321 \\
\hline Total or near-total & $368(67.4)$ & $6(85.7)$ & & $355(67)$ & 19 (82.6) & \\
\hline Subtotal & $64(11.7)$ & $1(14.3)$ & & $63(11.9)$ & $2(8.7)$ & \\
\hline Hemithyroidectomy & $114(20.9)$ & $0(0)$ & & $112(21.1)$ & $2(8.7)$ & \\
\hline Pathologic size (mm) & & & $<0.001$ & & & $<0.001$ \\
\hline Mean $\pm S D$ & $11.8 \pm 8.9$ & $29.4 \pm 19.5$ & & $11.5 \pm 8.6$ & $23.7 \pm 15.3$ & \\
\hline Median (interquartile range) & $10(5-15)$ & $30(11-40)$ & & $9(5-15)$ & $25(7-35)$ & \\
\hline Extrathyroidal extension & & & 0.137 & & & 0.060 \\
\hline No & $319(58.4)$ & $2(28.6)$ & & $312(58.9)$ & $9(39.1)$ & \\
\hline Yes & $227(41.6)$ & $5(71.4)$ & & $218(41.1)$ & $14(60.9)$ & \\
\hline Central lymph node metastasis & & & 0.476 & & & 0.675 \\
\hline No & $309(56.6)$ & $3(42.9)$ & & $300(56.6)$ & $12(52.2)$ & \\
\hline Yes & $237(43.4)$ & $4(57.1)$ & & $230(43.4)$ & $11(47.8)$ & \\
\hline Lateral lymph node metastasis & & & 0.189 & & & 0.039 \\
\hline No & $484(88.6)$ & $5(71.4)$ & & $472(89.1)$ & $17(73.9)$ & \\
\hline Yes & $62(11.4)$ & $2(28.6)$ & & $58(10.9)$ & $6(26.1)$ & \\
\hline Underlying echogenicity & & & 0.605 & & & 0.559 \\
\hline No & $457(83.7)$ & $7(100)$ & & $443(83.6)$ & $21(91.3)$ & \\
\hline Yes & $89(16.3)$ & $0(0)$ & & $87(16.4)$ & $2(8.7)$ & \\
\hline Multiplicity & & & 0.682 & & & 0.988 \\
\hline No & $402(73.6)$ & $6(85.7)$ & & $391(73.8)$ & $17(73.9)$ & \\
\hline Yes & $144(26.4)$ & $1(14.3)$ & & $139(26.2)$ & $6(26.1)$ & \\
\hline Radioactive iodine ablation & & & 0.712 & & & 0.951 \\
\hline No & $257(47.1)$ & $4(57.1)$ & & $250(47.2)$ & $11(47.8)$ & \\
\hline Yes & $289(52.9)$ & $3(42.9)$ & & $280(52.8)$ & $12(52.2)$ & \\
\hline Recurrence & & & 0.152 & & & 0.720 \\
\hline No & $492(90.1)$ & $5(71.4)$ & & $477(90)$ & $20(87)$ & \\
\hline Yes & $54(9.9)$ & $2(28.6)$ & & $53(10)$ & $3(13)$ & \\
\hline
\end{tabular}


on radioactive iodine WBS, which suggests regional or distant metastases. Patients with undetectable serum $\mathrm{Tg}$, with no regional recurrence on the neck US, with benign cytology results, and with no regional or distant metastasis on WBS or other imaging studies were considered to be disease free.

\section{Statistical Analysis}

Two different groups were formed using two different reference points of the cystic component, respectively. The two reference points were defined as a $25 \%$ and $50 \%$ cystic component because the percentage of cystic component of the thyroid nodules was visually categorized. Group 1 was divided by the reference point of a $50 \%$ cystic component. Group 2 was divided by the reference point of a $25 \%$ cystic component. All statistical analyses were carried out for both groups.

The chi-squared test or Fisher exact test was used to compare categorical variables. We compared continuous variables between the groups by using the independent 2-sample t-test. Disease-free survival (DFS) was defined as the time from the date of surgery to the date of death or the date of the last follow-up. Multivariate Cox proportional hazards regression models were used to identify whether a cystic component of more than $50 \%$ or $25 \%$ could be an independent predictor of recurrence during the follow-up period, after adjusting the known prognostic factors (tumor size, gender, age, extrathyroidal extension, and lymph node metastasis). To check the proportional hazards assumption, logs (-log[survival probability]) for different categories were plotted against time to ensure that the curves were reasonably parallel. All probability assumptions were appropriate. A P-value of less than 0.05 was considered statistically significant. Statistical analyses were performed with SAS ver. 9.1.3 (SAS Institute Inc., Cary, NC, USA).

\section{Results}

Table 2 summarizes the clinicopathologic features of the two groups. Fifty-six patients (10.1\%) were confirmed to have tumor recurrence within the follow-up period (Table 3). Recurrence in patients was diagnosed on the basis of cytopathologic results $(n=40)$, imaging features $(n=5)$ showing uptake in the neck area on WBS $(n=4)$, highly suspicious features of metastasis on cross-sectional imaging $(n=1)$, or biochemical recurrence showing elevated serum Tg levels $(n=12)$. Thirty-five patients had regional metastasis, one had distant metastasis, eight had multiple site metastases, and 12 had biochemical recurrence.

In group 1, thyroid cancers with a $>50 \%$ cystic component were significantly larger than those with a $\leq 50 \%$ cystic component in the case of the mean pathologic size $(P<0.001)$. There was a significant difference in gender $(P=0.047)$. The follow-up period ranged from 34 to 115 months (mean, $76.3 \pm 29.1$ months) for the 7 PTC patients with a $>50 \%$ cystic component and from 5 to 119 months (mean, 85.7 \pm 27.3 months) for the 546 patients with a $\leq 50 \%$ cystic component. Two recurrences occurred during the follow-up period in PTC patients with a $>50 \%$ cystic component $(28.6 \%$ ), whereas 54 recurrences occurred in patients with $\leq 50 \%$ cystic component (9.9\%) ( $\mathrm{P}=0.152)$. The cystic component of a tumor was not an independent predictor of recurrence (hazard ratio [HR], 1.118; 95\% confidence interval $[\mathrm{Cl}], 0.255$ to $4.910 ; \mathrm{P}=0.883$ ), according to the multiple Cox proportional hazards model (Table 4). Independent predictors of recurrence were pathologic size $(\mathrm{HR}, 1.035 ; 95 \% \mathrm{Cl}$, 1.008 to $1.064 ; P=0.012)$, male gender $(H R, 2.185 ; 95 \% \mathrm{Cl}, 1.093$ to 4.369; $P=0.027$ ), and lymph node metastasis ( $H R, 2.213 ; 95 \%$

Table 3. Demographic and clinicopathologic characteristics of 56 patients with recurrence

\begin{tabular}{lc}
\hline \multicolumn{1}{c}{ Characteristic } & No. (\%) \\
\hline Age (yr) & $45.6(24-79)$ \\
Mean (range) & $45.5(31.3-55)$ \\
Median (interquartile range) & $39: 17$ \\
Gender (female:male) & \\
Surgery & $47(83.9)$ \\
Total or near-total & $3(5.4)$ \\
Subtotal & $6(10.7)$ \\
Hemithyroidectomy & \\
Pathologic size (mm) & $18.5(2-65)$ \\
Mean size (range) & $13.5(7-27.3)$ \\
Median (interquartile range) & \\
Extrathyroidal extension & $19(33.9)$ \\
No & $33(58.9)$ \\
Perithyroidal & $1(1.8)$ \\
Strap muscle & $3(5.4)$ \\
Massive & $35(62.5)$ \\
Central lymph node metastasis & $22(39.3)$ \\
Lateral lymph node metastasis & $12(21.4)$ \\
Radioactive iodine ablation & \\
Yes & $24(14.3)$ \\
Ro & $31(55.4)$ \\
Biochemical recurrence & $25(44.6)$ \\
Opence & \\
Neck lymph nodion & \\
\hline
\end{tabular}


Table 4. Multivariate Cox proportional hazards regression analyses of variables predicting recurrence according to the cystic component

\begin{tabular}{lcccc}
\hline \multicolumn{1}{c}{ Variable } & $\mathrm{HR}(95 \% \mathrm{Cl})$ & P-value & HR $(95 \% \mathrm{Cl})$ & - \\
\hline$>50 \%$ cystic component & $1.118(0.255-4.910)$ & 0.883 & - & $0.569(0.164-1.976)$ \\
$>25 \%$ cystic component & - & - & $0.998(0.975-1.022)$ & 0.375 \\
Age & $0.998(0.975-1.022)$ & 0.857 & $1.040(1.012-1.070)$ & 0.884 \\
Pathologic size & $1.035(1.008-1.064)$ & 0.012 & $2.232(1.123-4.434)$ & 0.005 \\
Male gender & $2.185(1.093-4.369)$ & 0.027 & $1.535(0.954-2.469)$ & 0.022 \\
Extrathyroidal extension & $1.539(0.945-2.506)$ & 0.083 & $2.193(1.055-4.558)$ & 0.077 \\
Lymph node metastasis & $2.213(1.066-4.596)$ & 0.033 & 0.035 \\
\hline
\end{tabular}

$\mathrm{HR}$, hazard ratio; $\mathrm{Cl}$, confidence interval.

\section{$\mathrm{Cl}, 1.066$ to $4.596 ; \mathrm{P}=0.033)$.}

In group 2, thyroid cancers with a $>25 \%$ cystic component were also significantly larger than those with a $\leq 25 \%$ cystic component in the case of the mean pathologic size $(P<0.001)$. Lateral neck lymph node metastases were more frequently observed in thyroid cancers with a $>25 \%$ cystic component ( $P=0.039)$. The follow-up period ranged from 12 to 115 months (mean, $85.7 \pm 28.6$ months) for the 23 PTC patients with a $>25 \%$ cystic component and from 5 to 119 months (mean, $85.6 \pm 27.3$ months) for the 530 patients with a $\leq 25 \%$ cystic component. Three recurrences occurred during the follow-up period in PTC patients with a $>25 \%$ cystic component $(13 \%)$, whereas 53 recurrences occurred in those with a $\leq 25 \%$ cystic component (10\%; $P=0.720)$. The cystic component of a tumor was not an independent predictor of recurrence ( $H R, 0.569 ; 95 \%$ $\mathrm{Cl}, 0.164$ to $1.976 ; \mathrm{P}=0.375)$, using the multiple Cox proportional hazards model (Table 4). Independent predictors of recurrence were pathologic size (HR, 1.040; $95 \% \mathrm{Cl}, 1.012$ to $1.070 ; \mathrm{P}=0.005)$, male gender $(H R, 2.232 ; 95 \% \mathrm{Cl}, 1.123$ to $4.434 ; \mathrm{P}=0.022)$, and lymph node metastasis (HR, 2.193; $95 \% \mathrm{Cl}, 1.055$ to $4.558 ; \mathrm{P}=0.035$ ).

\section{Discussion}

PTC is the most common endocrine tumor arising from follicular cells of the thyroid, and its incidence has increased during recent years $[10,11]$. Most PTCs have an excellent prognosis, and factors known to be related to poor prognosis are age over 45 years, male gender, larger tumor size, presence of extrathyroidal invasion, or distant metastases at the time of diagnosis [12-19]. Mixed thyroid nodules, in which the tumor contains partially solid and cystic components, account for about $15.0 \%-53.8 \%$ of all thyroid nodules detected on US, and $17.6 \%$ of all cystic thyroid nodules are confirmed as malignant after thyroidectomy [8,20-23]. A predominant cystic change of thyroid cancer can be mistaken for a benign cystic hyperplastic nodule $[1,8,22,24]$. To distinguish benign nodules from malignancy in partially cystic thyroid nodules, US features such as eccentric configuration and presence of microcalcifications within the solid portion can be of help in an accurate diagnosis of thyroid malignancy [22,25-27]. Although US features and cytopathologic characteristics of cystic PTC have been established $[3,4,9,25,26]$, studies on PTC showing a cystic change on US focus on its behaviors, in comparison to solid-appearing thyroid cancers on US, and its prognosis has not yet been demonstrated. In this study, we investigated the nature of PTC with a cystic change on US. Measuring the component of cystic change of PTC on US is subjective, and calculation of the precise cystic portion on US is not always possible. Therefore, we divided the masses into two groups according to the cystic components by using two different reference points of $25 \%$ and $50 \%$, respectively.

In this study, only $1.3 \%$ of the patients diagnosed with PTC had masses containing more than a 50\% cystic component, and $4.2 \%$ had masses containing more than a $25 \%$ cystic component. Previously reported prevalence of cystic changes was $2.5 \%$ and $6 \%$ in patients with PTC $[1,2]$, and these percentages were higher than the values obtained in our study. To understand this difference, the process leading to a cystic change can be considered. The cystic change of a thyroid nodule may result from infarction or other destructive processes such as hemorrhage within the pre-existing follicles or cavities [28]. Nodule size may be affected by the grossly detectable cystic change, due to the presence of these follicles or cavities within the mass. The small percentage of PTC with cystic components in our study may be explained by the smaller size of PTC in our study than in other studies, since tumors of a smaller size are less likely to have areas of infarction or hemorrhagic cavities. Further, our study included nodules diagnosed as conventional PTC only, not other pathologies such as follicular thyroid carcinoma, medullary thyroid carcinoma, and undifferentiated thyroid carcinoma. This may have resulted in the lower percentage of thyroid cancer showing a cystic appearance in our study. 
Two recent studies have reported that the prognosis of cystic thyroid cancer is similar or better than conventional PTC $[29,30]$. One study reported that patients with cystic thyroid cancer have similar survival rates and clinical staging after receiving the same aggressive treatment, as compared to other solid and mixed thyroid cancer groups [29]. In that study, cystic thyroid cancer was defined as masses appearing as anechoic cysts on US images, and both papillary and follicular thyroid carcinomas were included. The other study reported that predominantly cystic cancers have more favorable prognoses and $95 \%$ of the patients were diseasefree on follow-up [30]. In this second study, pathologists evaluated morphologic features on pathology specimens, roughly categorizing them into cystic and marked cystic changes, and not according to the US images as in our study. In our study, we specified the cystic component according to its percentage on US, and included only nodules diagnosed as conventional PTC.

We tried to evaluate the association between the cystic change of PTC on US and the patient outcome. In the univariate analysis, age, gender, surgical method, and recurrence did not show significance between the two groups, namely groups 1 and 2 . However, the pathologic size was significantly larger in the group with more cystic components. There were no significant differences in DFS between the groups divided by cystic components. Moreover, a relatively high percentage of cystic components was not an independent predictor of recurrence after adjustment of other prognostic factors. The independent predictors of recurrence were pathologic size, male gender, and lymph node metastasis.

There were some limitations to our study. First, the percentage of cystic components was visually determined on the basis of US images by one radiologist, which is a subjective measurement method. Here, the limitation exists due to visual errors; a quantified classification may be more objective. Further, this study had a retrospective design, and the percentage of cystic components was determined by a retrospective review of US images and not during real-time examinations. Second, selection bias can exist when excluding patients for various reasons. This was inevitable, since this study did not focus on cystic PTC confirmed on pathology, but aimed to determine the relationship between the percentage of visible cystic components on US and patient prognosis by assessing the US features of nodules diagnosed as conventional PTC. Third, the small number of recurrences and PTC showing a cystic appearance on US make meaningful statistical comparisons difficult. Fourth, followup periods may not be sufficient when considering the favorable prognosis of PTCs as more cases might occur during a longer followup period.

In conclusion, the proportion of the cystic component in PTCs did not affect DFS in our study population.
ORCID: Ja Young Kim: http://orcid.org/0000-0002-7624-7259; Eun-Kyung Kim: http://orcid.org/0000-0002-3368-5013; Hye Sun Lee: http://orcid.org/0000-00016328-6948; Jin Young Kwak: http://orcid.org/0000-0002-6212-1495

\section{Conflict of Interest}

No potential conflict of interest relevant to this article was repoted.

\section{Acknowledgments}

This study was supported in part by the Research Fund of the Korean Society of Ultrasound in Medicine.

\section{References}

1. Henrichsen TL, Reading CC, Charboneau JW, Donovan DJ, Sebo TJ, Hay ID. Cystic change in thyroid carcinoma: prevalence and estimated volume in 360 carcinomas. J Clin Ultrasound 2010;38:361-366.

2. Chan BK, Desser TS, McDougall IR, Weigel RJ, Jeffrey RB Jr. Common and uncommon sonographic features of papillary thyroid carcinoma. J Ultrasound Med 2003;22:1083-1090.

3. Castro-Gomez L, Cordova-Ramirez S, Duarte-Torres R, Alonso de Ruiz P, Hurtado-Lopez LM. Cytologic criteria of cystic papillary carcinoma of the thyroid. Acta Cytol 2003;47:590-594.

4. Lin JD, Hsuen C, Chen JY, Liou MJ, Chao TC. Cystic change in thyroid cancer. ANZ J Surg 2007;77:450-454.

5. Abbas G, Heller KS, Khoynezhad A, Dubner S, Sznyter LA. The incidence of carcinoma in cytologically benign thyroid cysts. Surgery 2001;130:1035-1038.

6. Lin JD, Huang BY, Weng HF, Jeng LB, Hsueh C. Thyroid ultrasonography with fine-needle aspiration cytology for the diagnosis of thyroid cancer. J Clin Ultrasound 1997;25:111-118.

7. Muller N, Cooperberg PL, Suen KC, Thorson SC. Needle aspiration biopsy in cystic papillary carcinoma of the thyroid. AJR Am J Roentgenol 1985;144:251-253.

8. de los Santos ET, Keyhani-Rofagha S, Cunningham JJ, Mazzaferri EL. Cystic thyroid nodules. The dilemma of malignant lesions. Arch Intern Med 1990;150:1422-1427.

9. Yang GC, Stern CM, Messina AV. Cystic papillary thyroid carcinoma in fine needle aspiration may represent a subset of the encapsulated variant in WHO classification. Diagn Cytopathol 2010;38:721-726.

10. Rego-Iraeta A, Perez-Mendez LF, Mantinan B, Garcia-Mayor RV. Time trends for thyroid cancer in northwestern Spain: true rise in the incidence of micro and larger forms of papillary thyroid carcinoma. Thyroid 2009;19:333-340.

11. Davies L, Welch HG. Increasing incidence of thyroid cancer in the United States, 1973-2002. JAMA 2006;295:2164-2167.

12. Shaha AR. Implications of prognostic factors and risk groups in the management of differentiated thyroid cancer. Laryngoscope 
2004;114:393-402.

13. Shaha AR. Prognostic factors in papillary thyroid carcinoma and implications of large nodal metastasis. Surgery 2004;135:237-239.

14. Passler C, Scheuba C, Asari R, Kaczirek K, Kaserer K, Niederle B. Importance of tumour size in papillary and follicular thyroid cancer. Br J Surg 2005;92:184-189.

15. Machens A, Holzhausen HJ, Dralle H. The prognostic value of primary tumor size in papillary and follicular thyroid carcinoma. Cancer 2005;103:2269-2273.

16. Cady B. Hayes Martin Lecture. Our AMES is true: how an old concept still hits the mark: or, risk group assignment points the arrow to rational therapy selection in differentiated thyroid cancer. Am J Surg 1997;174:462-468.

17. Hay ID, Bergstralh EJ, Goellner JR, Ebersold JR, Grant CS. Predicting outcome in papillary thyroid carcinoma: development of a reliable prognostic scoring system in a cohort of 1779 patients surgically treated at one institution during 1940 through 1989. Surgery 1993;114:1050-1057.

18. Tennvall J, Biorklund A, Moller T, Ranstam J, Akerman M. Is the EORTC prognostic index of thyroid cancer valid in differentiated thyroid carcinoma? Retrospective multivariate analysis of differentiated thyroid carcinoma with long follow-up. Cancer 1986;57:1405-1414.

19. Byar DP, Green SB, Dor P, Williams ED, Colon J, van Gilse HA, et al. A prognostic index for thyroid carcinoma. A study of the E.O.R.T.C. Thyroid Cancer Cooperative Group. Eur J Cancer 1979;15:10331041.

20. Frates MC, Benson CB, Doubilet PM, Kunreuther E, Contreras M, Cibas ES, et al. Prevalence and distribution of carcinoma in patients with solitary and multiple thyroid nodules on sonography. J Clin Endocrinol Metab 2006;91:3411-3417.
21. Nam-Goong IS, Kim HY, Gong G, Lee HK, Hong SJ, Kim WB, et al. Ultrasonography-guided fine-needle aspiration of thyroid incidentaloma: correlation with pathological findings. Clin Endocrinol (Oxf) 2004;60:21-28.

22. Bellantone R, Lombardi CP, Raffaelli M, Traini E, De Crea C, Rossi $E D$, et al. Management of cystic or predominantly cystic thyroid nodules: the role of ultrasound-guided fine-needle aspiration biopsy. Thyroid 2004;14:43-47.

23. Rojeski MT, Gharib H. Nodular thyroid disease: evaluation and management. N Engl J Med 1985;313:428-436.

24. Hoang JK, Lee WK, Lee M, Johnson D, Farrell S. US Features of thyroid malignancy: pearls and pitfalls. Radiographics 2007;27:847860.

25. Kim DW, Lee EJ, In HS, Kim SJ. Sonographic differentiation of partially cystic thyroid nodules: a prospective study. AJNR Am J Neuroradiol 2010;31:1961-1966.

26. Lee MJ, Kim EK, Kwak JY, Kim MJ. Partially cystic thyroid nodules on ultrasound: probability of malignancy and sonographic differentiation. Thyroid 2009;19:341-346.

27. Hatabu H, Kasagi K, Yamamoto K, lida Y, Misaki T, Hidaka A, et al. Cystic papillary carcinoma of the thyroid gland: a new sonographic sign. Clin Radiol 1991;43:121-124.

28. Johannessen JV, Sobrinho-Simoes M. Follicular carcinoma of the human thyroid gland: an ultrastructural study with emphasis on scanning electron microscopy. Diagn Histopathol 1982;5:113-127.

29. Lin JD, Huang BY. Comparison of the results of diagnosis and treatment between solid and cystic well-differentiated thyroid carcinomas. Thyroid 1998;8:661-666.

30. Carcangiu ML, Zampi G, Rosai J. Papillary thyroid carcinoma: a study of its many morphologic expressions and clinical correlates. Pathol Annu 1985;20 Pt 1:1-44. 